\title{
The development of Ambulation Independence Measure: A new measurement tool to assess gait ability in acute stroke patients
}

\author{
Yusuke Hayashi ${ }^{\mathrm{a}, \mathrm{b}, *}$, Kota Yamazaki ${ }^{\mathrm{a}, \mathrm{b}}$, Kouichi Takeda ${ }^{\mathrm{a}, \mathrm{b}}$, Shujiro Ueda ${ }^{\mathrm{b}}$, Saiko Mikawa ${ }^{\mathrm{a}, \mathrm{b}}$, \\ Kozo Hatori $^{\mathrm{a}, \mathrm{b}}$, Kaoru Honaga ${ }^{\mathrm{a}}$, Tomokazu Takakura ${ }^{\mathrm{a}}$, Akito Hayashi ${ }^{\mathrm{a}, \mathrm{b}}$ and Toshiyuki Fujiwara ${ }^{\mathrm{a}, \mathrm{c}}$ \\ ${ }^{a}$ Department of Rehabilitation Medicine, Juntendo University Graduate School of Medicine, Tokyo, Japan \\ ${ }^{\mathrm{b}}$ Department of Rehabilitation Medicine, Juntendo University Urayasu Hospital, Chiba, Japan \\ ${ }^{\mathrm{c}}$ Department of Physical Therapy, Juntendo University Faculty of Health Science, Tokyo, Japan
}

Received 17 October 2021

Accepted 16 December 2021

\begin{abstract}
.
BACKGROUND: The assessment of gait function is important for stroke rehabilitation. Gait function of patients with stroke often depends on the type of orthosis. There are however few gait assessments that assess the type of orthosis.

OBJECTIVE: The purpose of this study was to investigate the reliability and validity of our newly developed Ambulation Independence Measure (AIM), which assesses the gait function, type of orthoses and physical assistance, for acute stroke patients.

METHODS: A total of 73 acute stroke patients participated in this prospective study. The AIM discriminates 7 levels of gait ability on the basis of the amount of physical assistance required and orthoses that are used during walking. Interrater reliability, concurrent validity with the Functional Ambulation Category (FAC) and predictive validity were examined.

RESULTS: The weighted kappas of AIM at the start of gait training (baseline) and discharge were 0.990 and 0.978 , respectively. The AIM scores were significantly correlated with the FAC scores at both baseline $(r=0.808)$ and discharge $(r=0.934)$. Multiple regression analyses showed that the AIM at baseline was a stronger predictor of the FAC at discharge $\left(\mathrm{R}^{2}=0.80\right)$.
\end{abstract}

CONCLUSIONS: The AIM has excellent reliability, concurrent validity, predictive validity, and good responsiveness in acute stroke patients.

Keywords: Cerebrovascular disorders, gait, orthosis, assessment, rehabilitation, stroke

\footnotetext{
*Address for correspondence: Yusuke Hayashi, MS, Department of Rehabilitation Medicine, Juntendo University Graduate School of Medicine 2-1-1 Hongo, Bunkyo-ku, Tokyo 113-8421, Japan. Tel.: +81 33813 3111; Fax: +81 33813 3111; E-mail: yusuke20100316@yahoo.co.jp.
}

\section{Introduction}

Stroke patients have various symptoms such as motor and sensory impairments. Their symptoms can cause gait disturbances, which have a negative effect on activities of daily living (ADL) and quality of life (QOL) (Patel AT et al., 2000, Reding MJ et al., 1988). 
Improving gait ability is one of the primary goals of stroke rehabilitation.

The use of a lower extremity orthosis, such as kneeankle-foot orthosis (KAFO) and ankle-foot orthosis (AFO), promotes active gait training and facilitates gait recovery (Maeshima S et al., 2017, Nikamp CDM et al., 2017, Ota T et al., 2018). A KAFO is usually prescribed when other forms of bracing (such as an AFO) are insufficient to adequately control knee instability due to hemiplegia (Fujii R et al., 2020, Hebert JS, 2006, Kakurai S et al., 1996, Maeshima S et al., 2017, Ota T et al., 2018, Yamanaka T et al., 2004). In acute stroke rehabilitation, KAFO are applied to the patients with severe hemiparesis for standing and gait training (Fujii R et al., 2020, Kakurai $S$ et al., 1996, Maeshima $S$ et al., 2017, Ota T et al., 2018, Yamanaka T et al., 2004). It is very difficult for patients to wear the KAFO in their ADL. For the patients with hemiparesis, it is necessary to change the KAFO to the AFO to acquire independent gait (Fujii R et al., 2020, Kakurai S et al., 1996, Maeshima S et al., 2017, Ota T et al., 2018, Yamanaka T et al., 2004).

The functional ambulation category (FAC) was developed to assess the gait ability of patients with stroke (Holden MK et al., 1986, Mehrholz J et al., 2007). The FAC distinguishes 6 levels of gait ability on the basis of the amount of physical assistance required (Holden MK et al., 1986, Mehrholz J et al., 2007). However, the FAC does not assess the types of orthoses. For example, the KAFO provides more stability for patients compared to the AFO (Boudarham J et al., 2013, Ota T et al., 2019). The amount of physical assistance with using the KAFO is less than the AFO even in same patient. Changing from KAFO to AFO for gait training is one of the favorable outcomes of acute stroke rehabilitation. Using an AFO results in an improved outcome but may result in the same FAC score. A more meaningful assessment of gait ability following a stroke includes an understanding of both how an AFO impacts gait and the level of physical assistance required.

Because of this, we developed a new measurement tool, the Ambulation Independence Measure (AIM), to assess the amount of physical assistance and the type of orthoses used. The conceptual basis of the AIM is as follows. To prevent overestimation of the participant's gait ability by lower limb orthoses (such as KAFO), AIM limit the types of lower limb orthoses and walking aids used during the walking trial to determine the AIM score, but FAC does not. In particular, during the walking trial to determine the AIM score, patients are allowed to use an AFO, crutch, or cane, but they are not allowed to use other orthoses or walking aids such as a KAFO, robotic device, parallel bar, or walker. The purpose of this study was to examine inter-rater reliability, concurrent validity, responsiveness, and predictive validity of the AIM in acute stroke patients.

\section{Methods}

\subsection{Ambulation independence measure (AIM)}

The AIM was developed with reference to the FAC (Holden MK et al., 1986, Mehrholz J et al., 2007). A FAC discriminates 6 levels (score range, 0-5) of gait ability on the basis of the amount of physical assistance required (see Table 1 for details).

The AIM discriminates 7 levels (score range, 1-7) of gait ability on the basis of the amount of physical assistance required. Patients are instructed to walk 5 meters, turn $180^{\circ}$, and walk back 5 meters. During the walking trial to determine the AIM score, patients are allowed to use an AFO, crutch, or cane, but they are not allowed to use other orthoses or walking aids such as a KAFO, robotic device, parallel bar, or walker. The definition of an AIM scoring is shown in Table 2.

The similarity between an AIM and a FAC is that the discriminant point of the evaluation of the amount of physical assistance is to support body weight or assist balance. The differences between an AIM and a FAC is that an AIM evaluates the participant's knee joint stability (the knee flexion angle in the paretic stance phase) during assisted walking, but FAC does not. To prevent overestimation of the participant's gait ability by lower limb orthoses (such as KAFO), an AIM limits the types of lower limb orthoses and walking aids during the walking trial to determine the AIM score, but FAC does not.

\subsection{Participants}

A prospective cohort study was conducted. Participants were recruited from among patients with an initial unilateral hemispheric stroke who were admitted to an acute hospital from March 2018 to March 2021. The diagnosis of stroke was based on the clinical history, neurologic examination, and head computed tomography or magnetic resonance imaging in each patient. A total of 198 patients matched the following inclusion criteria: 1) hemiparesis or hemiplegia, 2) gait disturbances (FAC score $<3$ ) and 3) 
Table 1

Functional ambulation category

\begin{tabular}{ll}
\hline Score & Gait ability \\
\hline 0 & A patient who is not able to walk at all or needs the help of two therapists \\
1 & A patient who requires continuous manual contact to support body weight as well as to \\
& maintain balance or to assist coordination \\
2 & A patient who requires intermittent or continuous light touch to assist balance or coordination \\
3 & A patient who can ambulate on level surface without manual contact of another person but \\
& requires standby guarding of one person either for safety or for verbal cueing \\
4 & A patient who can ambulate independently on level surface but requires supervision to \\
5 & negotiate (eg, stairs, inclines, non-level surfaces) \\
& A patient who can walk everywhere independently, including stairs \\
\hline
\end{tabular}

Table 2

Ambulation independence measure

\begin{tabular}{ll}
\hline Score & Gait ability \\
\hline 1 & A patient who is not able to walk with the physical assistance of one therapist using an AFO, \\
cane, or crutch \\
A patient who requires physical assistance to support body weight or maintain balance, but \\
shows severe knee buckling (the knee flexion angle in the paretic stance phase $\geq 30^{\circ}$ ) during \\
walking with an AFO, cane, or crutch \\
A patient who requires physical assistance to support body weight or maintain balance, and \\
shows mild to moderate knee buckling (the knee flexion angle in the paretic stance phase \\
$<$ \\
$<30^{\circ}$ ) during walking with an AFO, cane or crutch \\
4 & A patient who requires light touch to assist in balance using an AFO, cane or crutch \\
5 & A patient who can walk without manual contact by another person using an AFO, cane or \\
& crutch, but requires standby guarding by one person either for safety or for verbal cueing \\
6 & A patient who can walk independently using an AFO, cane, or crutch \\
7 & A patient who can walk independently without an AFO, cane, or crutch
\end{tabular}

AFO, Ankle Foot Orthosis.

under 90 years old. Patients were excluded if they had any of the following exclusion criteria: 1) unable to walk independently without a walking aid before onset (36 patients), 2) unable to follow instructions due to various symptoms such as severe aphasia and loss of consciousness (24 patients), 3) other medical complications or comorbidities that would alter the outcome of physical assessments (55 patients) and 4) unable to give consent to this study (10 patients). A total of 73 eligible patients agreed to participate. The protocol was approved by the Ethics Committee, and informed consent was obtained from all patients or their families before study participation.

All patients received conventional individual inpatient rehabilitation based on stroke rehabilitation guidelines, which involved gait training using a lower limb orthosis with human support by physical therapists (The Japan Stroke Society, 2015, Japanese Physical Therapy Association, 2017). The rehabilitation program included range of motion exercise, strengthening exercises, sitting balance exercise, standing balance exercises and gait training using a lower limb orthosis with manual assistance by physical therapists. The therapeutic time of physical therapy was ranged from $40-60 \mathrm{~min}$ according to the patient's physical status. The type of lower-limb orthosis used during gait training was determined clinically by the physical therapists depending on each patient's knee and ankle joint stability during gait (Kakurai S et al., 1996, Maeshima S et al., 2017, Ota $\mathrm{T}$ et al., 2018, Yamanaka T et al., 2004).

\subsection{Assessments}

Gait function was assessed using the AIM and the FAC at the start of gait training (baseline) and at discharge from the acute hospital to home or other facilities, such as rehabilitation hospitals. The type of lower-limb orthosis during the walking trial to determine the FAC score was the orthosis used in gait training. The type of lower-limb orthosis during the walking trial to determine the AIM score was the orthosis used in gait training, but when using KAFO, AFO was used instead of KAFO based on the measurement rules of an AIM. The walking aids during the walking trial determined clinically by the physical therapists and was defined as the use or no-use of a crutch or cane.

Individual deficits in lower-limb motor function, trunk function, and lower-limb sensory function were 
assessed at baseline. Lower extremity motor function was assessed using the lower extremity part of stroke impairment assessment set motor function (SIAS-M; score range, 0-15) (Chino N et al., 1994). Trunk function was assessed with the trunk impairment scale (TIS; score range, 0-21) (Fujiwara T et al., 2004). Lower extremity sensory function was assessed with the lower extremity part of SIAS sensory function (SIAS-S; score range, 0-6) (Chino $\mathrm{N}$ et al., 1994).

\subsection{Inter-rater reliability}

Two physical therapists assessed the AIM on the same day for patients at baseline and at discharge. Inter-rater reliability was examined using the weighted kappa statistics (Armitage P et al., 1994) and the Bland-Altman plots (Bland JM et al., 2012). According to Landis's classification, weighted kappa score of $0.81-1.0$ as almost perfect, $0.61-0.80$ as substantial, $0.41-0.60$ as moderate, $0.20-0.40$ as fair, and $<0.20$ as slight (Landis JR et al., 1977). Limits of agreement (LoA) were calculated as follows: mean difference between AIM scores (the AIM score minus the AIM score $) \pm(1.96 \times$ standard deviation $)$ (Giavarina D et al., 2015).

\subsection{Concurrent validity}

Concurrent validity was evaluated by Spearman's rank correlation coefficient (Armitage P et al., 1994) between the AIM scores and the FAC scores of patients at baseline and at discharge. The FAC was chosen because it has been proven to have high reli- ability and validity as a measure of gait performance (Holden MK et al., 1986).

\subsection{Responsiveness}

Responsiveness was assessed with standardized response means (SRMs). The SRM is the mean change in score divided by the standard deviation of the change scores (Landis JR et al., 1977). An SRM value $>0.80$ was considered large, $0.50-0.80$ moderate, and 0.2-0.5 small (Liang MH et al., 1990). Wilcoxon signed rank test (Armitage P et al., 1994) were used to assess the difference between baseline and discharge of the AIM and the FAC.

\subsection{Predictive validity}

Spearman's rank correlation coefficient (Armitage $P$ et al., 1994) and stepwise multiple regression analysis (Armitage P et al., 1994) was used to predict the FAC at discharge.

Statistical analysis was performed with SPSS version 24. $P$ values $<0.05$ were considered significant.

\section{Results}

Participants' characteristics at baseline are shown in Table 3. The mean age of participants was 64.9 (SD, 12.8) years. The mean time from stroke onset to baseline was 6.6 (SD 2.9) days. The mean length of stay in the acute hospital was 30.0 (SD 11.7) days. Table 4 shows gait ability at baseline and at discharge.

Table 3

Participants' characteristics

\begin{tabular}{lc}
\hline Age (yrs) & $64.9 \pm 12.8(39.0-88.0)$ \\
Sex (men/women) & $43 / 30$ \\
Stroke (ischemic/hemorrhagic) & $37 / 36$ \\
Side of lesion (left/right) & $43 / 30$ \\
\hline Time from onset to baseline (days) & $6.6 \pm 2.9(2.0-13.0)$ \\
Time from onset to discharge (days) & $30.0 \pm 11.7(13.0-70.0)$ \\
Time from baseline to discharge (days) & $24.3 \pm 11.7(10.0-66.0)$ \\
Discharge destination (inpatient rehabilitation & $66 / 7$ \\
facilities/home) & $39 / 34$ \\
\hline Orthoses user at baseline (KAFO/non-KAFO) & $24 / 49$ \\
Orthoses user at discharge (KAFO/non-KAFO) & $8.8 \pm 5.2(0.0-15.0)$ \\
\hline SIAS_M at baseline & $4.5 \pm 2.0(0.0-6.0)$ \\
SIAS_S at baseline & $17.0 \pm 4.5(4.0-21.0)$ \\
TIS at baseline &
\end{tabular}

Values are mean \pm SD (range) or number; KAFO, Knee Ankle Foot Orthosis; AFO, Ankle Foot Orthosis; SIAS-M, Lower-limb motor portions of Stroke Impairment Assessment Set; SIAS-S, Lower-limb sensory portions of Stroke Impairment Assessment Set; TIS, Trunk Impairment Scale. 


\subsection{Reliability}

The weighted kappas of the AIM at baseline and discharge were 0.990 (95\% confidence interval (CI), 0.970-1.009) and 0.978 (95\% CI, 0.952-1.004), respectively. These scores were classified as almost perfect using Landis's classification (Armitage $\mathrm{P}$ et al., 1994). The Bland-Altman plots indicated good agreement between the AIM scores at both baseline and discharge. At baseline, $1.37 \%$ (1/73) point was outside LoA $(-0.22 \sim 0.24)$. At discharge, $4.11 \%$ (3/73) point was outside LOA $(-0.50 \sim 0.61)$.

\subsection{Concurrent validity}

The AIM scores were significantly correlated with the FAC scores at both baseline $(r=0.808$, $P<0.0001)$ and discharge $(r=0.934, P<0.0001)$ (Tables 5 and 6).

In the 55 patients with an FAC score of 1 at baseline, the AIM score ranged from 1 to 3 . The patients with a FAC score of 1 at baseline were 36 KAFO users (65\%) and 19 non-KAFO users (35\%). All the patients with an AIM score of 1 or 2 at baseline were KAFO users. All the patients with an AIM score of 3 at baseline were non-KAFO users. In the 29 patients with an FAC score of 1 at discharge, the AIM score ranged from 1 to 3 . The patients with a FAC score of 1 at discharge were 14 KAFO users (48\%) and 15 non-KAFO users (52\%). All the patients with an AIM score of 1 or 2 at discharge were KAFO users. All the patients with an AIM score of 3 at discharge were non-KAFO users.

\subsection{Responsiveness}

The AIM and FAC scores changed significantly between baseline and discharge $(P<0.0001$ for both $)$ (Table 4). The SRMs of the AIM and the FAC were 1.396 and 1.056, respectively. The AIM and the FAC showed good responsiveness.

Table 4

Gait ability at baseline and discharge

\begin{tabular}{lccc}
\hline & Baseline & Discharge & $P$ value \\
\hline AIM (score, 1-7) & $2(1.0-3.5)$ & $4(2.0-6.0)$ & $<0.0001$ \\
FAC (score, 0-5) & $1(1.0-1.5)$ & $2(1.0-4.0)$ & $<0.0001$ \\
\hline
\end{tabular}

Values are median (quartile 1-quartile 3); $P$ values are the results of Wilcoxon signed rank test; AIM, Ambulation Independence Measure; FAC, Functional Ambulation Category.

\subsection{Predictive validity}

There was no significant correlation between the FAC at discharge and age $(r=-0.009, P=0.937)$ or time from baseline to discharge $(r=0.188, P=0.111)$. There was a significant correlation between the FAC at discharge and the AIM $(r=0.934, P<0.0001)$, FAC $(r=0.703, P<0.0001)$, SIAS-M $(r=0.811$, $P<0.0001)$, SIAS-S $(r=0.543, P<0.0001)$ or TIS $(r=0.682, P<0.0001)$ at baseline.

Multiple linear regression analyses were conducted using the AIM, FAC, SIAS-M, SIAS-S and TIS at baseline as predictor variables for determining the FAC at discharge, showed that $80 \%$ of the variance in the FAC at discharge was significantly independently predicted by the AIM $(\beta=0.606, p<0.001)$, TIS $(\beta=0.180, p=0.014)$ and SIAS-M $(\beta=0.202$, $p=0.037$ ) at baseline (Table 7).

\section{Discussion}

We developed the AIM to assess gait ability in the acute rehabilitation setting. This study examined the reliability and validity of the AIM.

In the most acute stroke patients, orthoses (such as $\mathrm{KAFO}$ and AFO) are used for gait training (Fujii R et al., 2020, Kakurai S et al., 1996, Maeshima S et al., 2017, Nikamp CDM et al., 2017, Ota T et al., 2018, Yamanaka T et al., 2004). Patients with hemiplegia are fitted with KAFOs for gait training (Fujii R et al., 2020, Kakurai S et al., 1996, Maeshima S et al., 2017, Ota T et al., 2018, Yamanaka T et al., 2004). As the patient's gait ability improves, KAFO will be changed to AFO (Fujii R et al., 2020, Kakurai S et al., 1996, Maeshima S et al., 2017, Ota T et al., 2018, Yamanaka $\mathrm{T}$ et al., 2004). Changing from a KAFO to an AFO means improved gait ability (Fujii R et al., 2020, Kakurai S et al., 1996, Hebert JS., 2006, Yamanaka T et al., 2004).

The FAC is a standard assessment for gait ability and assesses the amount of physical assistance for gait (Holden MK et al., 1986, Mehrholz J et al., 2007). The FAC, however, does not assess the type of orthosis (Holden MK et al., 1986, Mehrholz J et al., 2007). The physical assistance required for gait differ with the type of orthosis (Ota T et al., 2019, Hebert JS., 2006, Yamanaka T et al., 2004).

The AIM assesses physical assistance and the type of orthosis, and has limited walking aids and orthoses that can be used during walking trial. 
Table 5

Relationship between the ambulation independence measure scores and functional ambulation category scores at baseline

\begin{tabular}{lcccccccc}
\hline & \multicolumn{7}{c}{ AIM score } & Total \\
\cline { 2 - 7 } & 1 & 2 & 3 & 4 & 5 & 6 & 7 & \\
\hline FAC score 0 & 0 & 0 & 0 & 0 & 0 & 0 & 0 & 0 \\
FAC score 1 & 36 & 3 & 16 & 0 & 0 & 0 & 0 & 55 \\
FAC score 2 & 0 & 0 & 0 & 18 & 0 & 0 & 0 & 18 \\
FAC score 3 & 0 & 0 & 0 & 0 & 0 & 0 & 0 & 0 \\
FAC score 4 & 0 & 0 & 0 & 0 & 0 & 0 & 0 & 0 \\
FAC score 5 & 0 & 0 & 0 & 0 & 0 & 0 & 0 & 0 \\
\hline
\end{tabular}

Values are number; FAC, Functional Ambulation Category; AIM, Ambulation Independence Measure.

Table 6

Relationship between ambulation independence measure scores and functional ambulation category scores at discharge

\begin{tabular}{lcccccccc}
\hline & \multicolumn{7}{c}{ AIM score } & Total \\
\cline { 2 - 7 } & 1 & 2 & 3 & 4 & 5 & 6 & 7 & \\
\hline FAC score 0 & 0 & 0 & 0 & 0 & 0 & 0 & 0 & 0 \\
FAC score 1 & 14 & 8 & 7 & 0 & 0 & 0 & 0 & 29 \\
FAC score 2 & 2 & 0 & 1 & 6 & 0 & 0 & 0 & 9 \\
FAC score 3 & 0 & 0 & 0 & 0 & 14 & 0 & 0 & 14 \\
FAC score 4 & 0 & 0 & 0 & 0 & 0 & 5 & 6 & 11 \\
FAC score 5 & 0 & 0 & 0 & 0 & 0 & 1 & 9 & 10 \\
\hline
\end{tabular}

Values are number; FAC, Functional Ambulation Category; AIM, Ambulation Independence Measure.

Table 7

Results of stepwise multiple regression analysis to predict the gait ability at discharge

\begin{tabular}{lcccccc}
\hline $\begin{array}{l}\text { Dependent } \\
\text { variable }\end{array}$ & $\mathrm{R}^{2}$ & $\begin{array}{c}\mathrm{F}(P \\
\text { values })\end{array}$ & $\begin{array}{c}\text { Independent } \\
\text { variables }\end{array}$ & $\beta$ & $P$ value & VIF \\
\hline FAC at discharge & 0.80 & $96.3(<0.0001)$ & AIM at baseline & 0.606 & $<0.001$ & 2.658 \\
& & & TIS at baseline & 0.180 & 0.014 & 1.832 \\
& & & SIAS-M at baseline & 0.202 & 0.037 & 3.238 \\
\hline
\end{tabular}

$P$ values are the results of multiple regression analysis; FAC, Functional Ambulation Category; AIM, Ambulation Independence Measure; TIS, Trunk Impairment Scale; SIAS-M, Lower-limb motor portions of Stroke Impairment Assessment Set; VIF, Variance Inflation Factor.

The AIM showed excellent interrater reliability, concurrent validity, and predictive validity, and good responsiveness in acute stroke patients. The AIM score ranged from 1 to 3 in patients with an FAC score of 1 . The score of the AIM varied in patients with the same FAC score. It appears that the AIM reflects gait ability more accurately. This suggested that AIM is an effective measurement tool to assess gait ability in acute or subacute stroke rehabilitation.

The high interrater reliability of the AIM demonstrates that the clinical usefulness of the AIM in a clinical setting. The AIM assesses physical assistance needed to support body weight or maintain balance and buckling of the knee during gait with an AFO. That is the reason why it is easy for the physical therapist and physiatrist to evaluate the AIM.

The AIM score was significantly correlated with the FAC score at both baseline $(r=0.808, P<0.0001)$ and discharge $(r=0.934, P<0.0001)$. The FAC was significantly associated with many walking variables, such as gait speed and step length, showing good validity (Holden MK et al., 1986, Mehrholz J et al., 2007). The present results showed that the AIM has good concurrent validity. The patients with a severe gait disturbance (FAC score 1), however, were found to have AIM scores of 1,2, or 3. This difference can be explained by the type of orthoses used. The FAC assesses the amount of physical assistance, but not the type of orthoses used (Holden MK et al., 1986, Mehrholz J et al., 2007). Sixty-five percent of patients with an FAC score of 1 at baseline used KAFOs during gait training and evaluating the FAC. Our newly developed AIM assesses physical assistance and the type of orthosis. During the walking trial to determine the AIM score, patients are allowed to use an $\mathrm{AFO}$, crutch, or cane, but they are not allowed to use 
other braces or walking aids such as a KAFO, robotic device, parallel bar, or walker. All the patients with a FAC score of 1 and an AIM score of 1 or 2 used KAFO during gait training and evaluating the FAC. All the patients with a FAC score of 1 and an AIM score of 3 used AFO or no orthosis during gait training and evaluating the FAC. The use of a KAFO enhances a patient's knee joint stability (Boudarham $\mathrm{J}$ et al., 2013, Ota T et al., 2019) and may lead to the need for less physical assistance. This may account for the differences between FAC scores and AIM scores in patients with severe gait disturbances. The AIM can prevent overestimation of gait ability by the KAFO and can be used to obtain an accurate evaluation of gait ability for patients with a severe gait disturbance. In addition, the AIM might be helpful for selecting the orthosis suitable for gait training. For example, a KAFO can be applied for patients with an AIM score of 1 or 2, and an AFO or no brace can be applied for patients with an AIM score of 3 or higher.

Both the AIM and the FAC improved significantly from baseline to discharge. The SRMs of the AIM and FAC were 1.396 and 1.056 , respectively. The SRM is considered large if $>0.80$ (Crosby RD et al., 2003). These results indicate that the two measurements have satisfactory and comparable responsiveness.

Predictive validity is of key importance in outcome studies. Stepwise multiple regression analysis, which was performed with AIM, FAC, SIAS-M, SIAS-S, and TIS at baseline as the independent variables, showed that $80 \%$ of the variance in the FAC at discharge was significantly predicted by the AIM $(\beta=0.606, p<0.001)$, TIS $(\beta=0.180, p=0.014)$ and SIAS-M $(\beta=0.202, p=0.037)$. This suggested that the AIM has better predictive validity than the FAC, trunk function and lower extremity motor function. This result appears to support that the AIM more accurately reflects the gait ability of stroke patients with acute or severe gait disturbances than the FAC.

\subsection{Study limitation}

The primary limitations of this study were the difference in the time periods between the first and second assessments across patients, and the failure to consider the effects of treatment, including rehabilitation, during that time period. In particular, the time and content of gait training in rehabilitation may affect the improvement of gait ability. The content of the gait training, such as the type of the orthosis used, can differ depending on the gait ability. AIM enable to determine the gait ability for acute stroke patients. Therefore, by classifying acute stroke patients according to their gait ability and following up, the factors which affect the recovery of patient's gait ability can be examined in more detail.

\section{Conclusion}

We developed a new measurement tool, the AIM, to assess the amount of physical assistance and the type of orthoses used during the walking trial in acute stroke rehabilitation. The AIM has excellent interrater reliability, concurrent validity, and predictive validity, and good responsiveness in acute stroke patients. In particular, the AIM at the early onset was a strong predictor of gait ability at discharge, independent of the severity of hemiparesis or hemiplegia and trunk function. These findings suggest that the AIM is an effective measurement tool to assess gait ability and might be helpful for selecting the orthosis suitable for gait training in acute stroke rehabilitation, especially in patients with severe gait disturbances.

\section{Conflict of interest}

The authors report no conflicts of interest.

\section{Funding}

No funding was received for this article.

\section{References}

Armitage, P., \& Berry, G. (1994). Statistical Methods in Medical Research, ed 3. Oxford, Blackwell Scientific Publications.

Bland J. M., \& Altman D. G. (2012). Agreed statistics: measurement method comparison. Anesthesiology, 116 182-185.

Boudarham, J., Zory, R., Genet, F., Vigné, G., Bensmail, D., Roche, N., \& Pradon, D. (2013). Effects of a knee-ankle-foot orthosis on gait biomechanical characteristics of paretic and non-paretic limbs in hemiplegic patients with genu recurvatum. Clin Biomech, 28, 73-78.

Chino, N., Sonoda, S., Domen, K., Saitoh, E., \& Kimura, A. (1994). Stroke impairment assessment set (SIAS). Jpn J Rehabil Med, 31, 119-125.

Crosby, R. D., Kolotkin, R. L., \& Williams, G. R. (2003). Defining clinically meaningful change in health-related quality of life. J Clin Epidemiol, 56, 395-407.

Fujii, R., Sugawara, H., Ishikawa, M., \& Fujiwara, T. (2020). Effects of Different Orthoses Used for Gait Training on Gait Function among Patients with Subacute Stroke. Prog Rehabil Med, 5, 20200023. 
Fujiwara, T., Liu, M., Tsuji, T., Sonoda, S., Mizuno, K., Akaboshi, K., Hase, K., Masakado, Y., \& Chino, N. (2004). Development of a new measure to assess trunk impairment after stroke (trunk impairment scale): its psychometric properties. Am J Phys Med Rehabil, 83, 681-688.

Giavarina D. (2015). Understanding Bland Altman analysis. Biochem Med (Zagreb), 25, 141-151.

Hebert, J, S. (2006). Ambulatory KAFOs: a physiatry perspective. J Prosthet Orthot, 18, 169-174.

Holden, M. K., Gill, K. M., \& Magliozzi, M. R. (1986). Gait assessment for neurologically impaired patients. Standards for outcome assessment. Phys Ther, 66, 1530-1539.

Japanese Physical Therapy Association. (cited 2017 March 30). Japanese Guidelines for the Physical Therapy [internet]. Japan. Available from: http://www.japanpt.or.jp/upload/jspt/obj/files/guideline/12 _apoplexy.pdf.

Kakurai, S., \& Akai, M. (1996). Clinical experiences with a convertible thermoplastic knee-ankle-foot orthosis for post-stroke hemiplegic patients. Prosthet Orthot Int, 20, 191-194.

Landis, J. R., \& Koch, G. G. (1977). The measurement of observer agreement for categorical data. Biometrics, 33, 159-174.

Liang, M. H., Fossel, A. H., \& Larson, M. G. (1990). Comparisons of five health status instruments for orthopedic evaluation. Med Care, 28, 632-642.

Maeshima, S., Okamoto, S., Okazaki, H., Hiraoka, S., Funahashi, R., Yagihashi, K., Hori, H., Tanaka, S., Fuse, I., Asano, N., \& Sonoda, S. (2017). Lower limb orthotic therapy for stroke patients in a rehabilitation hospital and walking ability at discharge. Int J Phys Ther Rehabil, 3, 136.

Mehrholz, J., Wagner, K., \& Rutte, K. (2007). Predictive validity and responsiveness of the functional ambulation category in hemiparetic patients after stroke. Arch Phys Med Rehabil, 88, 1314-1319.
Nikamp, C. D. M., Hobbelink, M. S. H., van der Palen, J., Hermens, H. J., Rietman, J. S., \& Buurke, J. H. (2017). A randomized controlled trial on providing ankle-foot orthoses in patients with (sub-)acute stroke: short-term kinematic and spatiotemporal effects and effects of timing. Gait Posture, 55, 15-22.

Ota, T., Hashidate, H., Shimizu, \& N., Saito, A. (2018). Differences in activities of daily living between people with subacute stroke who received knee-ankle-foot and ankle-foot orthoses at admission. J Phys Ther Sci, 30, 1245-1250.

Ota, T., Hashidate, H., Shimizu, \& N., Saito, A. (2018). Difference in independent mobility improvement from admission to discharge between subacute stroke patients using knee-anklefoot and those using ankle-foot orthoses. J Phys Ther Sci, 30, 1003-1008.

Ota, T., Hashidate, H., Shimizu, \& N., Yatsunami, M. (2019). Early effects of a knee-ankle-foot orthosis on static standing balance in people with subacute stroke. J Phys Ther Sci, 31, 127-131.

Patel, A. T., Duncan, P. W., Lai, S. M., \& Studenski, S. (2000). The relation between impairments and functional outcomes poststroke. Arch Phys Med Rehabil, 81, 1357-1363.

Reding, M. J., \& Potes, E. (1988). Rehabilitation outcome following initial unilateral hemispheric stroke. Life table analysis approach. Stroke, 19, 1354-1358.

The Japan Stroke Society, editors. (2015). Japanese Guidelines for the Management of Stroke. KYOWA KIKAKU, Tokyo.

Yamanaka, T., Akashi, K., \& Ishii, M. (2004). Stroke rehabilitation and long leg brace. Top Stroke Rehabil, 11, 6-8. 